\title{
Cost of cleanliness may sink European Mars mission
}

London. The European Space Agency (ESA) says that it may not be able to afford a mission to Mars unless an international committee lowers standards intended to protect other planets from contamination from Earth. The ESA budget is so tight that the cost of sterilization regimens and other measures needed to prevent Earth organisms from reaching the planet could reduce the scientific payload or the number of probes; either result would make it harder for the Mars mission to compete against other possible ESA missions.

International guidelines have changed several times since the Viking probes landed on Mars in 1976. COSPAR (the Committee on Space Research), organized by the International Council of Scientific Unions, which has responsibility for setting standards of planetary protection, is expected to relax the rules further when it meets later this month at the World Space Congress in Washington, DC. But the change may not be enough for ESA's Marsnet.

Marsnet, one of four proposals being mission, would land three physically unprobes on the martian surface to study seismic and atmospheric conditions. The design incorporates as few protection measures as possible; essentially, it provides that the probes will be as clean as possible, but that only surfaces that will come into direct contact with Mars need to be sterilized.

Spending money on further protective measures will reduce the amount of science that can be done and will rule out a possible fourth probe. Although scientists have not determined an exact price, a full bioshield such as that used by Viking would breach considered for ESA's next medium-sized connected but functionally complementary

the \$300-million ceiling set for mediumsized missions

Marsnet is the only candidate mission involving a planetary landing. Of the others, PRISMA is a helio-seismology project, INTEGRAL is a gamma-ray astronomy project and STEP is a fundamental physics project that will test the principle of equivalence. A decision will be made next June, with a tentative launch in 2001.

Complementary to Marsnet is a proposed mission by the US National Aeronautics and Space Administration (NASA), called MESUR, to land between eight and twentythree probes on the martian surface. Although it is subject to the same COSPAR regulations as ESA, MESUR features protection similar to that used on Viking.

The Viking mission searched for life on Mars: apart from anxiety about introducing Earth organisms to Mars, researchers had to be sure that any life forms detected by the Viking probes did not come from Earth. Each probe was sealed in a box before the launch and baked at $112^{\circ} \mathrm{C}$ for 40 hours. According to NASA's John Rummel, the cost of the planetary protection is not a factor in the MESUR project.

The first of the Mars landing missions, assuming the money is available, will be the Russian Mars 94 project. It is not known just what planetary protection measures the Russians are planning, but they have promised to abide by COSPAR rules. The mission consists of a large orbiter with a very heavy scientific payload and two landers, which may penetrate the surface.

The consensus view is that Mars, believed to be the only extraterrestrial planet capable of supporting life (indigenous or otherwise), needs special protection. Even

\section{EC asked to supplement Framework}

Munich. Filippo Pandolfi, head of the European Commission (EC) research programmes, has asked for an extra ECU1.6 billion (US $\$ 2.15$ billion) to continue research and development projects now that a delay in the next five-year programme, due to start in 1993, seems inevitable. The funds would be used to support the EC's current Framework research plan, which ends in 1994, and to ensure that funding for applied research does not dry up. No change in funding of biomedical research is expected, and there will be no expansion of the human capital and mobility schemes.

The next programme is expected to be a victim of increasing bureaucracy within the commission. A legal challenge earlier this if the scientific aims are not primarily exobiological, there are good arguments for keeping up standards of planetary protection. If a probe discovered a site with a temperature profile, water content or level of oxidation that would be friendly to life, its findings could be thrown into doubt by the question of contamination. On a larger scale, organisms deposited on the surface may be spread over a large area by the martian dust storms.

Although these possibilities are considered remote, delegates to the COSPAR meeting are expected to exercise caution. A panel of the US National Academy of Sciences is completing a study commissioned by NASA that Rummel expects will recommend looser requirements for probes landing on the martian surface but continued caution about those going beneath the surface.

Less is known about conditions beneath the martian surface, but the thinking is that they might be similar to those around Earth volcanoes. Any evidence collected by the proposed missions that demonstrates life-supporting conditions on Mars will once again raise standards for planetary protection.

lan Mundell

\section{ICI splits in two, forms drugs company}

London. The British conglomerate Imperial Chemical Industries (ICI) unveiled plans last week to put most of its research-intensive divisions into a separate company. Pharmaceuticals, agrochemicals, seeds and specialty chemicals would join to form ICI Bioscience, while the company's other interests - paints, materials, industrial chemicals, explosives and surfactants - will remain as ICI. This demerger, as it is being called by ICI, is expected to be confirmed at a meeting of shareholders early in 1993.

In 1991, ICI as a whole spent $£ 692$ million (US\$1.3 billion) on research, of which 70 per cent was carried out by the divisions that will go to form ICI Bioscience. With current revenues of $£ 3.9$ billion, ICI Bioscience would become the world's ninth largest drugs group, although commentators have noted that much of its sales comes from agrochemicals. The remaining bulkchemicals company would drop from fifth to seventh in the world.

The financial and the scientific communities welcomed the news: the consensus is that a company focused on research will be better able to refill a pharmaceutical pipeline drying up over the next three to four years. Frank Kaye, a senior partner in PA Consulting, an international science consultancy, points out that the demerger combines all the genuinely global parts of ICI. "Pharmaceuticals and agrochemicals can get on with being international businesses now that they are away from the anglocentricity of the bulk chemicals divisions", he says. Ian Mundell 